\title{
Pediatric Acute Toxic Leukoencephalopathy: Prediction of the Clinical Outcome by FLAIR and DWI for Various Etiologies
}

\author{
(D). Ozturk, (D). Rykken, and (D) A.M. McKinney
}

\begin{abstract}
BACKGROUND AND PURPOSE: Pediatric acute toxic leukoencephalopathy is a clinicoradiologic entity comprising various etiologies. This study aimed to identify the MR imaging appearance of pediatric acute toxic leukoencephalopathy from various etiologies and determine whether the etiology correlates with clinical outcome.
\end{abstract}

MATERIALS AND METHODS: We retrospectively reviewed the electronic records of patients with pediatric acute toxic leukoencephalopathy younger than 19 years of age who had MR imaging within $<2$ weeks of presentation, including DWI and FLAIR sequences. Two neuroradiologists scored the DWI and FLAIR severity and measured the percentage ADC reduction within the visibly affected regions and normal-appearing WM. The percentage ADC reduction and DWI and FLAIR severity were correlated with clinical outcome using the Spearman correlation.

RESULTS: Of 22 children, 3 were excluded due to a nontoxic cause or incomplete examination. Regarding the included 19 children (mean age, 13 years), the etiologies of pediatric acute toxic leukoencephalopathy were the following: methotrexate $(n=6)$, bone marrow transplantation $(n=4)$, fludarabine $(n=3)$, cytarabine $(n=1)$, carboplatin $(n=1)$, vincristine $(n=7)$, cyclosporine $(n=1)$, uremia $(n=1)$, and bevacizumab $(n=1)$. Three subgroups were analyzed (chemotherapy, $n=12$; immunosuppression, $n=5$; others, $n=2)$. There was a strong correlation of FLAIR $(r=0.773, P<.001)$ and DWI $(r=0.851, P<.001)$ severity with clinical outcome, and patients treated with fludarabine had the worst outcomes. High percentage ADC reduction values were associated with adverse outcomes, and lower percentage ADC reduction values were associated with favorable outcomes $(r=0.570, P=.011)$.

CONCLUSIONS: The DWI and FLAIR severity scores appear highly prognostic, whereas percentage ADC reduction is moderately prognostic for clinical outcomes in pediatric acute toxic leukoencephalopathy. Immunosuppressive pediatric acute toxic leukoencephalopathy tends toward favorable outcomes, and fludarabine tends toward worse outcomes.

ABBREVIATIONS: \%ADCR = percentage ADC reduction; ATL = acute toxic leukoencephalopathy; BMT = bone marrow transplantation; NAWM = normalappearing WM; PATL = pediatric acute toxic leukoencephalopathy; PRES = posterior reversible encephalopathy; PVWM = periventricular WM

$\mathbf{T}$ he term "toxic leukoencephalopathy" involves a broad spectrum of etiologies that may produce structural injuries to the WM; the insults may be acute or chronic and arise secondary to chemotherapy or immunosuppressive therapy, environmental toxins, or infectious etiologies. ${ }^{1,2}$ The clinical features of acute toxic leukoencephalopathy (ATL) can be variable but are typically abrupt in onset and based on the extent of involved areas in the brain parenchyma; these symptoms range from minimal cognitive deficits to severe neurologic impairment. ${ }^{3}$ Recognition of this

Received March 19, 2020; accepted after revision April 30.

From the Department of Radiology, University of Minnesota, Minneapolis, Minnesota.

Please address correspondence to Kerem Ozturk, MD, Division of Neuroradiology, Department of Radiology, University of Minnesota, B-226 Mayo Memorial Building, MMC 292,420 Delaware St SE, Minneapolis, MN 55455; e-mail: oztur027@umn.edu http://dx.doi.org/10.3174/ajnr.A6624 disorder is important because the clinical and imaging findings may reverse abruptly after removal of the underlying cause but the outcome may also be fatal. ${ }^{4}$ In children or infants, pediatric ATL (PATL) may occur due to various factors and may, in theory, be characterized by typical neurologic deficits with distinct MR imaging findings, compared with ATL affecting adults. ${ }^{4,5}$ However, only sporadic cases of PATL have been reported previously. ${ }^{6}$

Numerous distinct toxic etiologies have been reported to cause PATL, such as chemotherapeutic and immunosuppressive agents, antimicrobials, environmental toxins, bone marrow transplantation (BMT), and others. ${ }^{7,8}$ However, the differential causes of PATL need to be expanded, and it is unknown whether there are differences in their imaging severity or whether such differences reflect their outcomes. For example, preliminary efforts in adult ATL have shown that immunosuppressive medication- 
related ATL has more favorable outcomes relative to both chemotherapy- and opiate-related ATL and that the MR imaging severity may correlate with outcome in certain subgroups. ${ }^{9,10}$ Hence, the combination of qualitative and quantitative MR imaging features could aid in predicting the clinical outcomes of PATL among different etiologies because DWI can reveal brain injury in PATL at an early stage, particularly within the periventricular WM (PVWM). ${ }^{11-15}$ Hence, because FLAIR and DWI are usually regarded as routine sequences for brain imaging, these sequences could be beneficial in determining characteristic regions of involvement and severity of injury in PATL. ${ }^{11}$

Hence, this study describes a cohort of children and infants having PATL secondary to various toxic insults who presented with acute neurologic deficits. Considering that prior studies have been limited primarily to case reports, the purpose of this larger study was to determine the differences in the DWI and FLAIR appearances of PATL according to various etiologies, to measure their severity on DWI and FLAIR, and to assess their clinical outcomes using both ADC values and MR imaging severity scores.

\section{MATERIALS AND METHODS Study Design and Participants}

This retrospective study was approved by the institutional review board of a tertiary care center that specializes in pediatric oncology and organ transplantation. All procedures performed were in accordance with the ethical standards of the institutional research committee and with the 2013 revised Declaration of Helsinki and its later amendments or comparable ethical standards. Informed consent was not required. Children with suspected PATL were identified via the PACS archives between January 2000 and October 2019. Children were included if the clinical presentation was acute neurologic deterioration with an MR imaging within 2 weeks of presentation, including DWI (with ADC maps) and FLAIR sequences. The MRIs of children suspected of having PATL were reviewed and graded by 2 neuroradiologists. The clinical and laboratory records were reviewed for each child to identify the etiology of PATL, and patients were excluded if the clinical impression did not indicate PATL (an imaging-clinical discordance), the MR imaging did not show PATL, the patient was older than 18 years of age, or there were other causes of PVWM-reduced diffusion (eg, infectious, hypoxic, congenitalmetabolic, or an isolated callosal splenium lesion [reversible cytotoxicity]). ${ }^{12}$ Because overlapping etiologies occasionally cause both PATL and posterior reversible encephalopathy syndrome (PRES), the reviewers ensured that there was a lack of cortical edema, often an initial manifestation of PRES. ${ }^{13,14}$

\section{MR Imaging Technique}

During the 19 years of this study, multiple $1.5 \mathrm{~T}$ and 3T MR imaging units were used, so the sequence parameters varied slightly. Spin-echo T1WI, T2WI, and FLAIR images were obtained for all patients. The MR imaging parameters varied with time and field strength; however, at 1.5T, the FLAIR parameters were the following: TR range/TE range, 6500-9000/105-110 ms; TI, 2000-2100 ms; number of excitations, 1-2; echo-train length, 15-23. The DWI parameters were TR/TE range, 3300-4000/71-120 ms. At
3T, the parameters for FLAIR were the following: TR/TE range, 9000-11,000/100-120 ms; TI, 2000-2100 ms; number of excitations/echo-train length, $1-2 / 10-25 \mathrm{~ms}$, respectively. For DWI, the parameters were TR/TE range, 2800-3000/70-90 ms. The gradient strength was $b=1000 \mathrm{~s} / \mathrm{mm}^{2}$ for DWI at $1.5 \mathrm{~T}$ and 3T. The section thickness was typically always $5 \mathrm{~mm}$. For all DWI sequences, ADC maps were obtained.

\section{MR Imaging Severity Scoring, ADC Measurements, and Clinical Outcome}

A staff neuroradiologist (A.M.M.) and a neuroradiology fellow (K.O.), each blinded to the clinical outcome and etiologies of PATL, independently reviewed all MR images. Each study was assessed at least twice during separate sessions. In patients in whom there were different interpretations, the scans were re-evaluated and a consensus was reached. The reviewers graded the DWI and FLAIR severity via a previously described system, ranging from grades 0 to $4(0=$ normal, $4=$ severe $){ }^{13-15}$

The DWI and ADC maps were visually assessed for abnormalities, and ADC values within the ROIs were evaluated; the ROIs were identified on the original T2WI and visually matched on the $b=0$ DWI. The ADC values were obtained using a mean of 3 values of $>5$-mm circular ROIs from the affected PVWM (according to visual assessment of the maximal area of involvement); similar measurements were acquired from the nonaffected WM (normalappearing WM [NAWM]). If the findings of PATL were bilateral, the ROIs were averaged between both sides. The ROIs from the NAWM were typically obtained from deep frontal or temporal WM. Thereafter, the percentage of reduction in ADC (\%ADCR) was calculated by a ratio of the ADC measurement in the affected WM to that of the NAWM. ${ }^{16,17}$ All ADC measurements were obtained by the neuroradiology fellow separately before the MR imaging severity scoring.

The tabulated clinical outcomes were based on a retrospective evaluation of the patients' records and the ATL outcome score (based on a clinical follow-up of $>30$ days). ${ }^{15}$ The outcomes of this scale were the following: 0 , complete resolution of acute symptoms, without residual abnormalities on the neurologic examination; 1, moderately improved neurologic examination, with minimal residual neurologic deficit relative to initially; 2 , mildly improved neurologic examination with moderate residual deficit; 3 , spastic quadriplegia or profound developmental delay; 4 , coma or death.

\section{Statistical Analysis}

A paired Student $t$ test was used to compare the ADC measurements from the affected WM with the NAWM. The \%ADCR, DWI severity score, and FLAIR severity score were individually correlated using Spearman rank correlation coefficients, with 2-sided $P$ values. Thereafter, the \%ADCR and DWI and FLAIR severity scores were each correlated with the clinical outcomes. A correlation $(r)$ of $0.0-0.2$ was considered a minimal or negligible correlation; $>0.2-0.4$, a mild correlation; $>0.4-0.7$, a moderate correlation; and $>0.7-1.0$, a strong correlation. To statistically compare among subgroups, we compared the PATL subcategory etiologies using a Kruskal-Wallis test for continuous variables, the Fisher exact test for categoric variables, and a pair-wise comparison 
Table 1: Brain MR imaging abnormalities, their distribution, locations of reduced diffusion, size of the largest WM lesion, \%ADCR values, and clinical outcomes

\begin{tabular}{|c|c|c|c|c|c|c|c|c|c|c|c|}
\hline No. & $\begin{array}{l}\text { Age } \\
\text { (yr) }\end{array}$ & Sex & Size $(\mathrm{cm})$ & $\begin{array}{c}\text { Underlying } \\
\text { Dx }\end{array}$ & Etiologies & Groups & $\begin{array}{l}\text { Regions of Red. } \\
\text { Diffusion }\end{array}$ & $\begin{array}{c}\text { FLAIR } \\
\text { Severity }\end{array}$ & $\begin{array}{c}\text { DWI } \\
\text { Severity }\end{array}$ & $\begin{array}{l}\text { \%ADC } \\
\text { Red. }\end{array}$ & Outcome \\
\hline 1 & 16 & $M$ & $4.2 \times 2.2$ & $\overline{A L L}$ & Methotrexate & $C$ & $\mathrm{Fr}, \mathrm{CS} / \mathrm{CR}$ & 1 & 1 & 0.32 & 1 \\
\hline 2 & 14 & M & $2.1 \times 0.8$ & ALL & Methotrexate & $\mathrm{C}$ & $\mathrm{Fr}, \mathrm{CS} / \mathrm{CR}$ & 1 & 1 & 0.16 & 0 \\
\hline 3 & 18 & M & $4.5 \times 2.6$ & ALL & Methotrexate & $\mathrm{C}$ & $\mathrm{Fr}+\mathrm{P}, \mathrm{CS} / \mathrm{CR}$ & 1 & 2 & 0.39 & 2 \\
\hline 4 & 16 & $\mathrm{~F}$ & $2.1 \times 1.8$ & ALL & Cytarabine & $\mathrm{C}$ & $\mathrm{Fr}, \mathrm{CS} / \mathrm{CR}$ & 1 & 1 & 0.35 & 2 \\
\hline 5 & 11 & M & $4.5 \times 2.2$ & ALL & Methotrexate & $\mathrm{C}$ & $\mathrm{Fr}, \mathrm{CS} / \mathrm{CR}, \mathrm{CC}$ & 0 & 2 & 0.36 & 2 \\
\hline 6 & 11 & $\mathrm{~F}$ & $4.1 \times 1.8$ & ALL & Methotrexate & C & $\mathrm{Fr}+\mathrm{P}, \mathrm{CS} / \mathrm{CR}$ & 1 & 2 & 0.41 & 2 \\
\hline 7 & 14 & $\mathrm{~F}$ & D & AML & Fludarabine & $\mathrm{C}$ & $\mathrm{D}, \mathrm{CS} / \mathrm{CR}, \mathrm{CC}, \mathrm{Th}$ & 4 & 4 & 0.32 & 3 \\
\hline 8 & 17 & $\mathrm{~F}$ & $2.3 \times 0.4$ & ALL & Methotrexate & $\mathrm{C}$ & $\mathrm{Fr}+\mathrm{P}, \mathrm{CS} / \mathrm{CR}$ & 2 & 2 & 0.32 & 3 \\
\hline 9 & 1 & $\mathrm{~F}$ & $3.8 \times 1.1$ & ATRT & Carboplatin & $\mathrm{C}$ & $\mathrm{Fr}+\mathrm{P}, \mathrm{CS} / \mathrm{CR}, \mathrm{Th}$ & 2 & 3 & 0.57 & 4 \\
\hline 10 & 15 & $\mathrm{~F}$ & $2.4 \times 1.4$ & PTLD & Methotrexate & $\mathrm{C}$ & $\mathrm{Fr}+\mathrm{P}, \mathrm{CS} / \mathrm{CR}, \mathrm{BS}, \mathrm{CC}$ & 3 & 3 & 0.29 & 4 \\
\hline 11 & 10 & M & D & $\mathrm{AML}$ & Fludarabine & C & $\mathrm{D}, \mathrm{CS} / \mathrm{CR}, \mathrm{Th}, \mathrm{BS}$ & 4 & 4 & 0.62 & 4 \\
\hline 12 & 17 & $\mathrm{~F}$ & $5.4 \times 2.0$ & AML & Fludarabine & C & $\mathrm{Fr}+\mathrm{P}, \mathrm{CS} / \mathrm{CR}$ & 2 & 2 & 0.48 & 4 \\
\hline 13 & 13 & $\mathrm{~F}$ & $1.5 \times 0.7$ & JMML & BMT & I & $\mathrm{Fr}, \mathrm{CS} / \mathrm{CR}$ & 1 & 1 & 0.17 & 0 \\
\hline 14 & 16 & $\mathrm{~F}$ & $2.8 \times 0.8$ & $\mathrm{AA}$ & BMT & I & $\mathrm{Fr}, \mathrm{CS} / \mathrm{CR}$ & 1 & 1 & 0.42 & 0 \\
\hline 15 & 17 & M & $1.3 \times 1.1$ & AML & Cyclosporine & I & $\mathrm{P}, \mathrm{CS} / \mathrm{CR}$ & 1 & 1 & 0.06 & 1 \\
\hline 16 & 17 & $\mathrm{~F}$ & 0 & AML & BMT & I & NA & 1 & 0 & 0.02 & 0 \\
\hline 17 & 1 & M & $2.8 \times 0.5$ & RMS & BMT & I & $\mathrm{Fr}, \mathrm{CS} / \mathrm{CR}$ & 1 & 1 & 0.15 & 2 \\
\hline 18 & 7 & M & $3.9 \times 1.9$ & ATRT & Bevacizumab & $\mathrm{O}$ & $\mathrm{Fr}, \mathrm{CS} / \mathrm{CR}$ & 1 & 1 & 0.51 & 2 \\
\hline 19 & 16 & M & $7.9 \times 2.4$ & ESRD & Uremia & 0 & $\mathrm{Fr}+\mathrm{P}, \mathrm{CS} / \mathrm{CR}$ & 2 & 2 & 0.57 & 3 \\
\hline
\end{tabular}

Note:-AA indicates aplastic anemia; ALL, acute lymphocytic leukemia; AML, acute myeloid leukemia; ATRT, atypical teratoid/rhabdoid tumor; \%ADC red., percentage ADC reduction; $B S$, brain stem; CC, corpus callosum; $C R$, corona radiata; CS, centrum semiovale; $D$, diffuse; Dx, diagnosis; ESRD, end-stage renal disease; Fr, frontal; JMML, juvenile myelomonocytic leukemia; NA, not applicable; P, parietal; PTLD, post-transplant lymphoproliferative disorder; RMS, rhabdomyosarcoma; Th, thalami.

for subgroup-subgroup analysis to assess significant differences in FLAIR/DWI severity or outcomes among subgroups. For each test, a value of $P<.05$ was considered statistically significant. The statistical analysis was performed via commercially available statistical software (SPSS, Version 23.0; IBM).

\section{RESULTS}

\section{Patient Demographics and Etiologies}

Within the 19-year period of this review, 25 children presented with an appearance of PVWM and isolated corpus callosumreduced diffusion on the initial MR imaging, eventually confirmed clinically to be related to ATL. Of these, 6 were excluded due to clinical or imaging impressions that did not favor PATL (ie, clinical-imaging discordance, $n=1$ ), the MR imaging findings did not suggest PATL (predominantly cortical edema suggesting PRES, $n=2$ ), or reversible callosal splenial lesions $(n=3)$. The 3 with reversible splenial lesions were excluded because that entity is not typically categorized as ATL, in which the acute presentation and limited lesion extent have a benign clinical prognosis. ${ }^{12}$ Ultimately, a total of 19 children (10 female; mean age, 13 years; age range, $1-18$ years) met the inclusion criteria and served as the final cohort.

The etiologies of PATL were methotrexate $(n=7)$, marrow transplantation (without definite medication identified, $n=4$ ), fludarabine $(n=3)$, cytarabine $(n=1)$, carboplatin $(n=1)$, cyclosporine $(n=1)$, bevacizumab $(n=1)$, and uremia $(n=1)$. For statistical analysis, the etiologies were categorized into 3 subgroups: chemotherapy (group C) $(n=12)$; immunosuppression (group I, including patients with transplantation and immunosuppression) $(n=5)$; and others (group O) $(n=2)$. Table 1 provides the causes, sites, and sizes of reduced diffusion; etiologies; imaging grade; and the clinical outcome of each child with PATL.

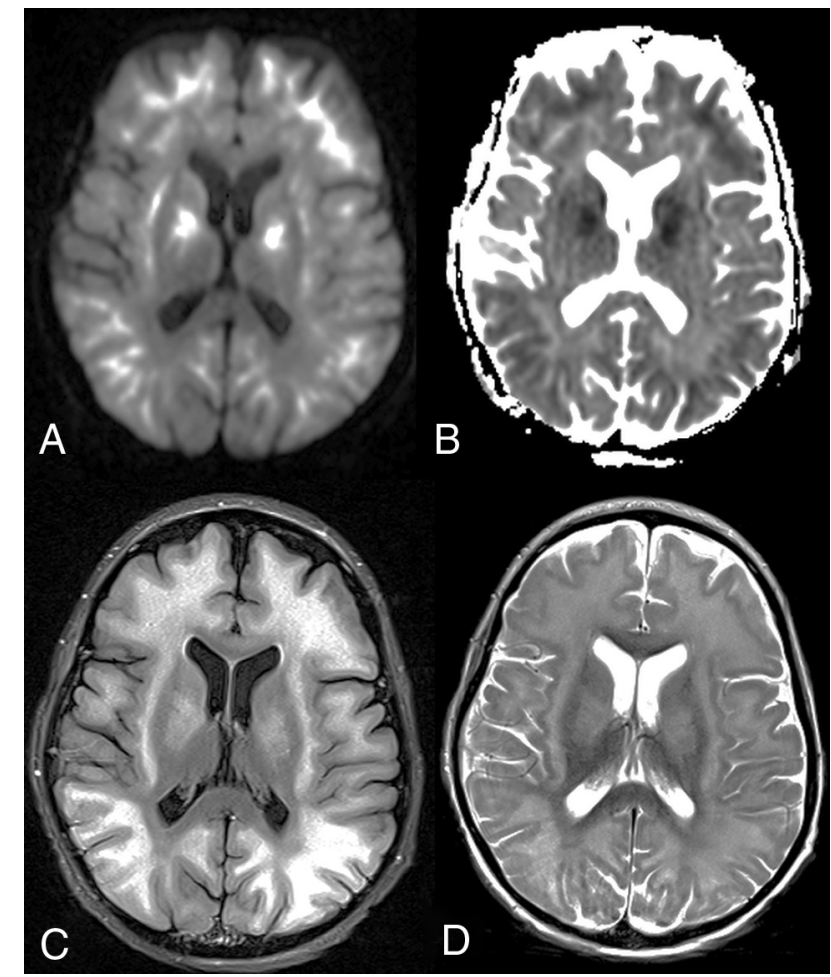

FIG 1. A 10-year-old boy with fludarabine-related PATL (patient 11). Axial DWI $(A)$ and ADC map $(B)$ show symmetric, extensive, and confluent diffusely reduced diffusion with the corresponding hyperintensity on axial FLAIR $(C)$ and T2WI $(D)$ in the subcortical WM and basal ganglia. The child died 7 days after the insult, with the ATL outcome score being 4 (ie, severe outcome).

The age and sex were not correlated with the DWI/FLAIR severity and \%ADCR (all, $P>.05$ ). Samples of children's MR images are shown in Figs 1 and 2. 


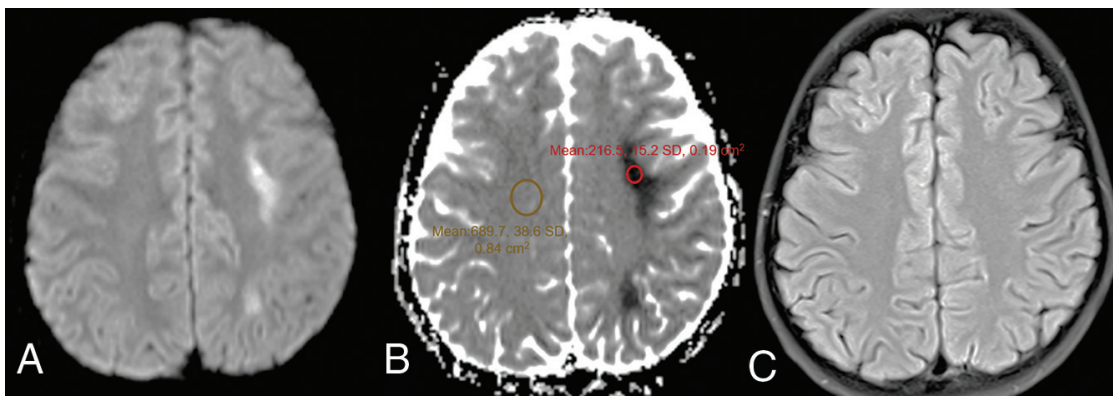

FIG 2. An 11-year-old boy with methotrexate-induced PATL (patient 5). Axial DWI (A) and ADC map (B) show unilateral reduced diffusion within the deep left frontoparietal PVWM without significant corresponding hyperintensity on an axial FLAIR image (C). The patient had moderate long-term neurologic sequelae, with the ATL outcome score at 1 month being 2. An ADC map (B) illustrates the placement of ROls for the measurement of ADC values, as well as to determine the \%ADCR. Such ROls were located in the affected WM lesion (red ROI) and nonaffected WM (NAWM) (orange ROI).

Table 2: Mean demographics, imaging characteristics, and clinical outcomes according to different subgroups of PATL

\begin{tabular}{lcccc}
\hline \multicolumn{1}{c}{ Characteristics } & Group C $(\boldsymbol{n}=12)$ & Group I $(\boldsymbol{n}=5)$ & Group O $(\boldsymbol{n}=2)$ & Total $(\boldsymbol{n}=19)$ \\
\hline Sex & & & & \\
Male (No.) & 5 & 2 & 2 & 9 \\
Female (No.) & 7 & 3 & 0 & 10 \\
Age (yr) & 13.3 & 12.8 & 11.5 & 13 \\
FLAIR severity score & 1.8 & 1 & 1.5 & 1.5 \\
DWI severity score & 2.25 & 0.8 & 1.5 & 1.78 \\
\%ADCR & 0.38 & 0.16 & 0.54 & 0.34 \\
Clinical outcome $^{\mathrm{a}}$ & 2.58 & 0.6 & 2.5 & 2.05 \\
\hline
\end{tabular}

${ }^{a}$ Four of 19 patients (21\%) did not have a $>30$-day clinical outcome available.

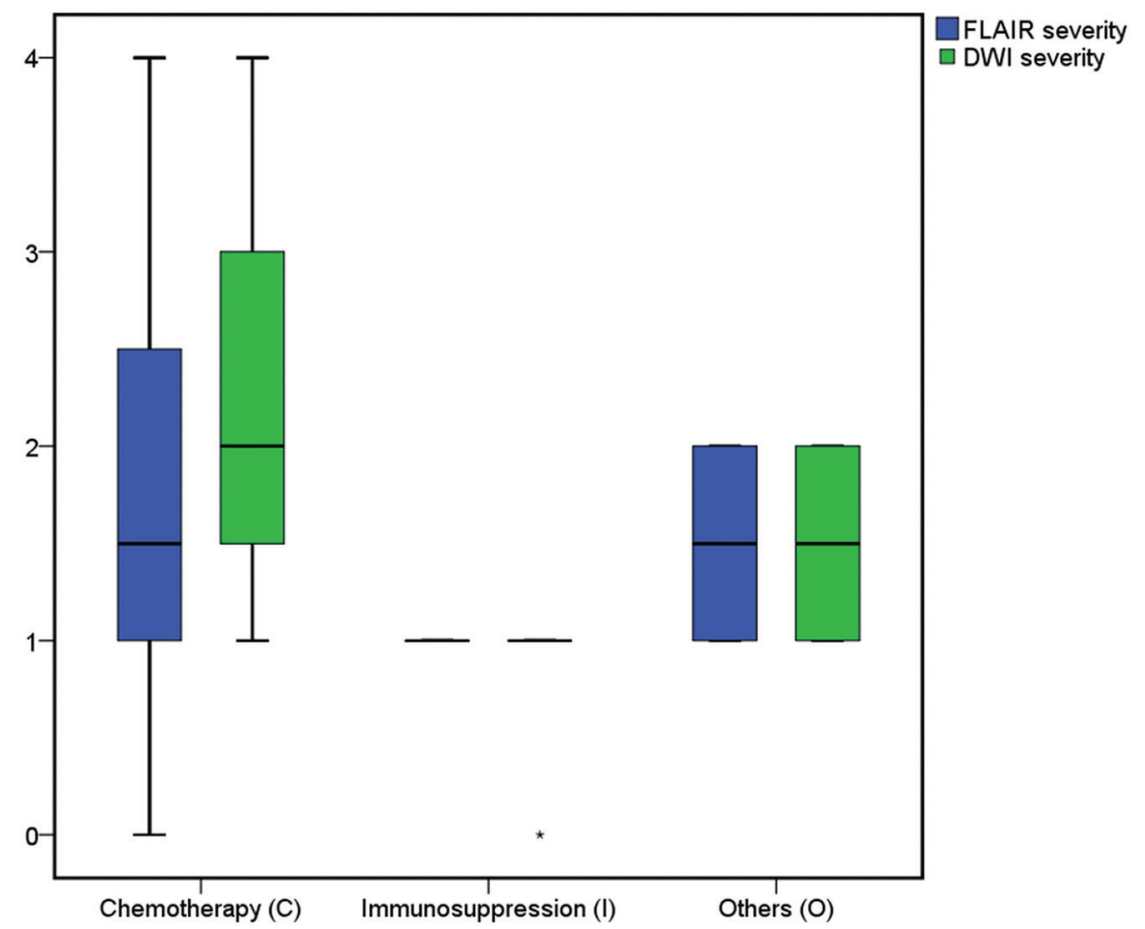

FIG 3. A boxplot demonstrates the mean FLAIR and DWI severity scores for PATL according to the different subgroups that caused PATL.

\section{DWI and FLAIR Severity}

Table 2 provides the mean DWI and FLAIR severity scores. In 4/19 children (21\%), the WM lesions on FLAIR were revealed, which showed corresponding reduced diffusion on DWI. Overall, there were significant differences between the mean DWI and FLAIR severity scores among the 3 major groups $(P<.05)$ : Group I had lower mean DWI severity compared with group $C$ and group $\mathrm{O}$ (DWI severity, $P=.008$ ). Children on chemotherapeutic agents and those with other etiologies had similar DWI and FLAIR severity scores $(P>.05)$. Figure 3 shows the mean FLAIR and DWI severity.

\section{Percentage ADC Reduction}

The mean ADC values for the entire cohort of 19 patients with PATL are listed in Tables 1 and 2, with the $\% A D C R$ measured in the affected PVWM relative to the NAWM. In these 19 children, a significant difference was seen between the ADC values in the affected PVWM versus the NAWM $(P<.001$, paired Student $t$ test). In particular, groups $\mathrm{C}$ and $\mathrm{O}$ had significantly higher \%ADCR values compared with group I $(P<.05)$, while children with chemotherapeutic agents and those from other etiologies had similar \%ADCR values $(P>.05)$. A boxplot (Fig 4) demonstrates that the lowest \% ADCR was in the affected PVWM for group I (mean, 16.4\%), while the highest was in group O (mean, 54\%).

\section{Clinical Outcome}

Table 2 provides the mean clinical outcome score for each of the 3 subgroups. Of note, 4 of 19 patients (21\%) did not have a $>30$-day clinical outcome available. Overall, there were significant differences among the 3 major subgroups for the clinical outcome scores $(P<.05)$. When we compared the outcomes of these 3 subgroups against each other, groups $\mathrm{C}$ and $\mathrm{O}$ had significantly worse clinical outcomes compared with group I $(P=.009)$, while patients treated with chemotherapy and those from other etiologies had similar outcomes $(P>.05)$. Notably, severe outcomes (grade 4) were noted in 4/19 (21\%) 


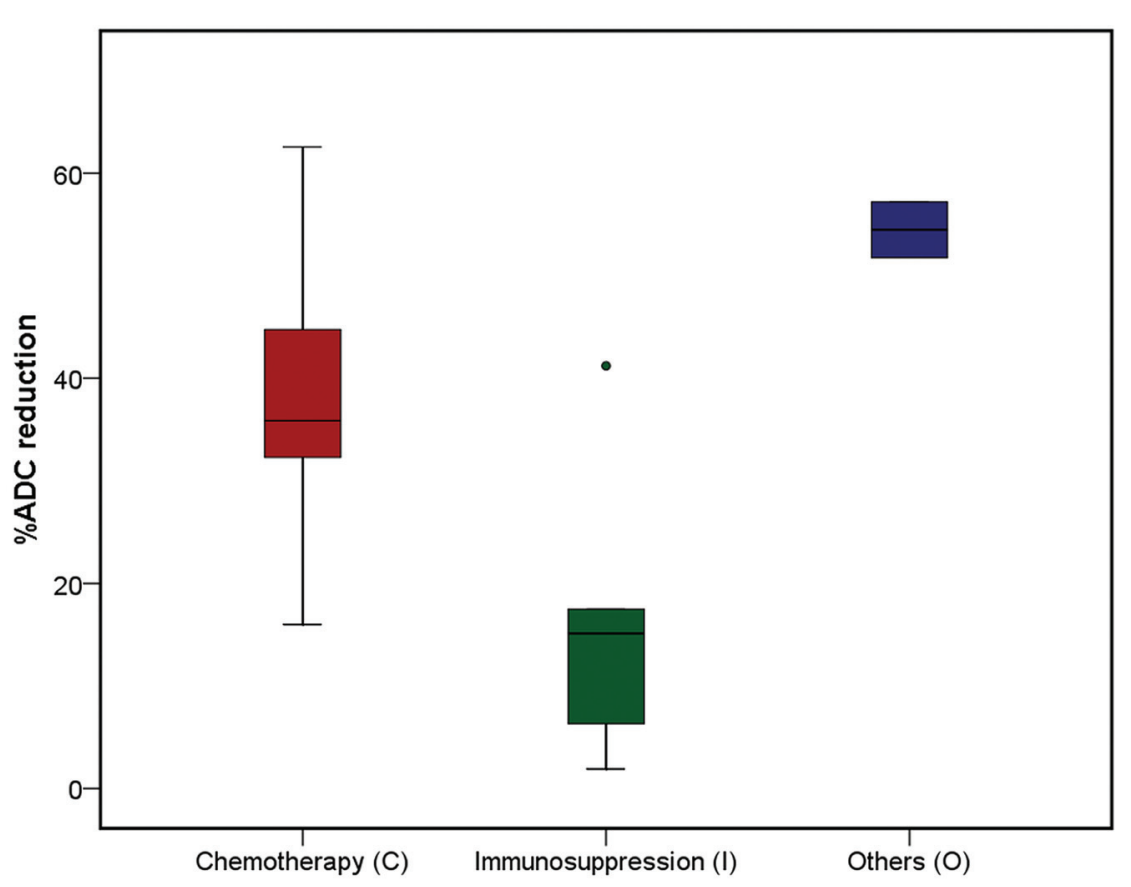

FIG 4. A boxplot of each etiologic group with the \%ADCR of the affected PVWM regions compared with NAWM. Notably, the subgroup of PATL related to chemotherapy had a significantly greater \%ADCR compared with the immunosuppression group. PATL from other causes (group O) demonstrated a trend toward greater \%ADCR compared with immunosuppression group, but without reaching statistical significance.

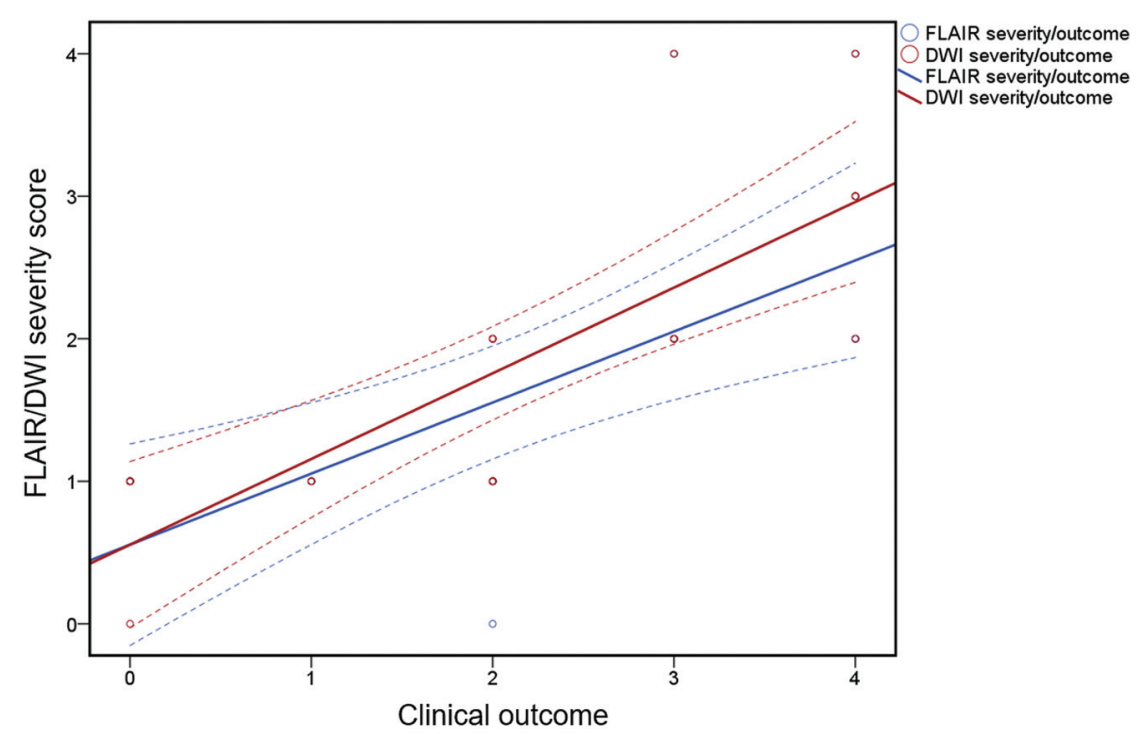

FIG 5. A scatterplot and regression line, which together display the positive relationship between the FLAIR/DWI severity scores and clinical outcome.

patients with available data, 2 of which were related to fludarabine; of those 4 with severe outcomes, 2 died and 2 were comatose at 30 days. In contrast, there was either symptom resolution or only mild sequelae (outcome scores, $0-1$ ) in 6/19 (31.5\%) of those with PATL, with $9 / 19$ (47.3\%) having moderate-severe sequelae (ie, scores 2-3). The clinical outcome was overall less severe for immunosuppressive-related PATL.
Correlation of MR Imaging Findings with Clinical Outcome

Overall, there was a strong correlation between the DWI and FLAIR severity scores with the clinical outcome ( $r=0.851, P<.001$ for DWI severity; $r=0.773, P<.001$ for FLAIR severity), as shown in Fig 5, with a moderate correlation between the \%ADCR and clinical outcome $(r=0.570, P=.011)$. The scatterplot in Fig 6 has a regression line that shows a positive relationship between the \%ADCR and clinical outcomes. Regarding the 3 subgroups of etiologies of PATL, both the DWI and FLAIR severity correlated strongly with clinical outcome for group $\mathrm{C}$ ( $r=0.787, P=.002$ for DWI severity; $r=0.789, P=.002$ for FLAIR severity), but no significant correlation existed for the other 2 subgroups (each $P>.05)$. There was no significant correlation of \%ADCR with clinical outcome via intergroup comparison (each $P>.05)$. Also, neither age nor sex correlated with the DWI or FLAIR severity, nor with the \%ADCR (all $P>.05$ ).

\section{DISCUSSION}

PATL is an uncommon clinicoradiologic diagnosis characterized by cerebral WM injury on DWI. This entity should be considered in the differential diagnosis of a child who presents with a recent onset of neurologic deficit and a known exposure to an external toxin, such as medications (most common in this cohort). ${ }^{5}$ Recognition of this condition is crucial because it is potentially reversible on prompt removal of the underlying etiology with supportive treatment. However, the clinical outcome varies between the etiologies, as found in the current study. Of note, prior studies of adult ATL have similarly found that a heterogeneous variety of causes has been defined that can lead to toxic leukoencephalopathy with similar clinical and imaging presentations. ${ }^{10,15}$ Thus, this study may be the first to both describe a common appearance of PATL on DWI and FLAIR shared by such various entities and assess whether DWI and FLAIR differ in outcome.

Thus, this study attempted to determine variations in the DWI and FLAIR imaging appearances, to evaluate the severity of PATL in these different etiologies, and to assess their clinical outcomes using both DWI and FLAIR severity scoring. Thus, higher 


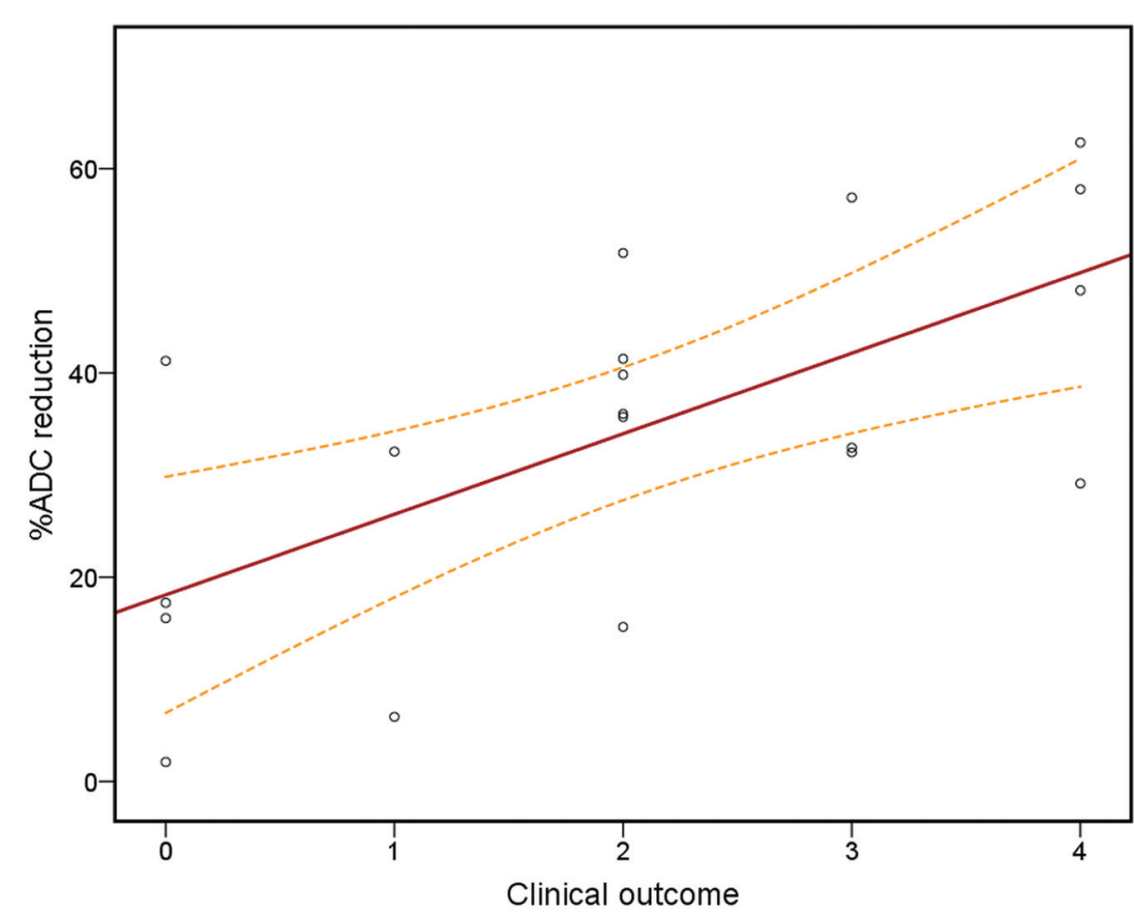

FIG 6. A scatterplot and regression line, which together display the positive relationship between the \%ADCR and clinical outcome.

\%ADCR values were found to be associated with adverse outcomes, and strong correlations were observed between the FLAIR and DWI severity with clinical outcome. Such observations likely highlight the significance of discerning early cases of PATL to initiate prompt therapy. Thus, the presence of a correlation between the MR imaging findings and clinical outcome suggests that the MR imaging severity may reflect the prognosis in PATL. This possibility suggests that there may be several important reasons to identify the early MR imaging findings of PATL in children presenting with acute symptoms. First, a minority of these patients continue to deteriorate; hence, the patients may deteriorate and even die after persistent exposure to a toxic agent, as described previously with chemotherapeutic agents. ${ }^{18,19}$ Second, PATL may present with rather nonspecific-but-severe symptoms and thus can be confused clinically with other irreversible syndromes (eg, hypoxic-ischemic encephalopathy or PRES); consequently, the window for effective treatment could be missed. ${ }^{20}$ Third, prompt recognition of PATL can prevent inappropriate treatment with potential iatrogenic complications in patients suspected of having another or underlying disorder.

Many etiologies have been documented anecdotally to cause PATL, including a variety of chemotherapeutic and immunosuppressive medications, BMT, and perhaps other etiologies such as uremia and sepsis. ${ }^{4,10}$ Accordingly, in this larger study, half of the cases of PATL were caused by chemotherapy, with methotrexate being the most common (37\%), as well as fludarabine (16\%). Regarding these etiologies, the current study attempted to attribute etiologies to determine whether they have different outcomes in PATL. Overall, the DWI and FLAIR severity scoring appears prognostic, while the \%ADCR was only moderately prognostic in predicting outcomes. Notably, fludarabine-related PATL had the worst clinical outcome, being the most common cause of death from PATL, in accordance with prior results in adults. $^{3,21}$ Thus, in PATL, the presence of characteristic regions of involvement on DWI and FLAIR, combined with quantitative ADC analysis in the appropriate clinical setting, may allow early diagnosis and potentially help determine the prognosis in children with PATL. $^{18}$

Regarding intergroup comparisons of outcome, the DWI and FLAIR severity was worse in chemotherapy-related PATL, which also had worse clinical outcomes, despite the potentially reversible nature of PATL. In comparison, immunosuppression-related PATL had milder MR imaging severity and better clinical outcomes than the other subgroups, in accord with a recent study of adult ATL. ${ }^{11}$ Thus, chemotherapy-related PATL having relatively strong correlations between the imaging severity and outcome suggests that more diffuse brain involvement in PATL tends to result in neurologic sequelae.

The basic pathophysiologic mechanisms leading to PATL and reduced diffusion are unknown but are likely multifactorial, being thought related to BBB dysfunction and endothelial injury. For example, chemotherapeutic agents such as methotrexate and fludarabine have been shown to directly injure the microvasculature and can also have indirect excitotoxic effects. ${ }^{22}$ Meanwhile, immunosuppressive medications, such as tacrolimus, cyclosporine, and mycophenolate are thought to cause capillary endothelial injury, leading to BBB dysfunction and occasional WM injury; thus, such injury from these agents may manifest as PRES (more commonly) or ATL (less commonly) based on prior studies. ${ }^{3,13,23}$ Hence, the cause of reduced diffusion in the PVWM in PATL likely varies by etiology but may arise from intramyelinic edema, cytotoxicity via capillary endothelial injury, and/or direct toxic demyelination. Accordingly, histopathologic studies have corroborated such insults by discovering WM injury with axonal and oligodendroglial swelling, and also macrophage infiltration. ${ }^{1,15}$

Limited studies used DWI in children with PATL, being mostly anecdotal. ${ }^{4,24,25}$ DWI is more sensitive than T2WI or FLAIR in depicting the abnormalities of PATL; DWI can also help to determine the age of lesions, such as whether they are acute. ${ }^{26}$ Another benefit of DWI is the ability to measure quantitative ADC values; thus, pathologic foci on DWI can be objectively measured on the ADC maps to exclude T2 shine through effects, which can mimic the reduced diffusion of acute lesions. ${ }^{27}$

The main limitation of this study is its retrospective nature. For example, the clinical outcomes were based on the retrospective assessment of the medical record, in which long-term followup was not available in some patients; thus, the relatively small number of patients from a single tertiary care center may limit 
the number of etiologies evaluated and thus potentially affect the outcome analysis. Another limitation is that PATL is becoming slowly-but-increasingly recognized during the past 15-20 years; therefore, the frequency of this disorder may not be as uncommon as reported in this study, perhaps being underreported. Additionally, serial imaging was not performed routinely in the current study; therefore, the potential reversibility or progression of the brain injury could not be entirely assessed in each patient.

Another potential limitation is the differences in the physiologic MR imaging signal of WM structures between the pediatric and adult brain that may result in differences in the MR imaging findings and clinical outcomes between pediatric and adult ATL. ${ }^{28}$ For example, during the first year of life, WM signal intensity decreases with time on T2WI via the physiologic process of myelination, which is mostly completed in the second year of life. To prevent this possible confounding factor, children younger than 12 months of age were not included in the study. This is largely due to the difficulty in measuring the effects of WM maturation on MR imaging signal intensity within the first 2 years, when the modeling of these variables would be highly complex; hence, a statistical correction cannot be readily applied to quantify the effects of PATL in infants.

Another possible limitation is the differing MR imaging scanner field strengths, with some children being imaged on a $1.5 \mathrm{~T}$ scanner and others at 3T; notably, the differences in appearances and ADC values between these field strengths were not evaluated in this study. Nevertheless, this scenario mimics what often happens in routine clinical practice across centers and is also a consequence of the retrospective nature of this study. Most interesting, some authors have observed no statistically significant differences in ADC values comparing various regions of PVWM between magnetic field strengths (1.5 and 3T), so it is uncertain whether this observation would affect the results of the current study. ${ }^{15}$ Hence, this is another reason that the relative ADC measurements of affected WM (compared with NAWM) were used rather than using absolute ADC measurements. Hence, a prospective study is needed that incorporates control patients, obtains PATLs at regular intervals with a single MR imaging scanner, and implements advanced MR imaging applications such as DTI, to confirm the findings of our study of affected regions based on visual inspection. ${ }^{29}$

\section{CONCLUSIONS}

The DWI and FLAIR MR imaging severity scores appear highly prognostic of clinical outcomes in PATL, while the \%ADCR seems to be only moderately prognostic. Of note, chemotherapyrelated PATL (in particular, when related to fludarabine) overall had worse outcomes, while immunosuppression-related PATL had better outcomes. Thus, characteristic regions of involvement on DWI and FLAIR imaging as well as quantitative ADC analyses in the appropriate clinical setting may allow early diagnosis and possibly the determination of prognosis of PATL, but these need to be evaluated by a larger, prospective study.

Disclosures: Alexander M. McKinney-UNRELATED: Board Membership: Veeva Systems, Inc, Comments: board member and CEO, Veeva Systems, Inc (Minneapolis,
Minnesota), an Informatics solutions company; Employment: University of Minnesota, Fairview.

\section{REFERENCES}

1. Rimkus Cde M, Andrade CS, Leite Cda C, et al. Toxic leukoencephalopathies, including drug, medication, environmental, and radiation-induced encephalopathic syndromes. Semin Ultrasound CT MR 2014;35:97-117 CrossRef Medline

2. Koksel Y, Ozutemiz C, Rykken J, et al. "CHOICES": an acronym to aid in delineating potential causes of non-metabolic, non-infectious acute toxic leukoencephalopathy. Eur J Radiol Open 2019;6:243-57 CrossRef Medline

3. Beitinjaneh A, McKinney AM, Cao Q, et al. Toxic leukoencephalopathy following fludarabine-associated hematopoietic cell transplantation. Biol Blood Marrow Transplant 2011;17:300-08 CrossRef Medline

4. Luckman J, Zahavi A, Efrati S, et al. Difficulty in distinguishing posterior reversible encephalopathy syndrome, hypoxic-ischemic insult, and acute toxic leukoencephalopathy in children. Neuropediatrics 2016;47:33-38 CrossRef Medline

5. Kontzialis M, Huisman T. Toxic-metabolic neurologic disorders in children: a neuroimaging review. J Neuroimaging 2018;28:587-95 CrossRef Medline

6. De Cauwer H, De Wolf P, Couvreur F, et al. An unusual case of 4aminopyridine toxicity in a multiple sclerosis patient: epileptic disorder or toxic encephalopathy? Acta Neurol Belg 2009;109:40-41 Medline

7. Abeyakoon O, Batty R, Mordekar S, et al. The encephalopathic child. Neuroradiol J 2011;24:483-502 CrossRef Medline

8. Kumar Y, Drumsta D, Mangla M, et al. Toxins in brain! magnetic resonance (MR) imaging of toxic leukoencephalopathy: a pictorial essay. Pol J Radiol 2017;82:311-19 CrossRef Medline

9. Okumura A, Kidokoro H, Tsuji T, et al. Differences of clinical manifestations according to the patterns of brain lesions in acute encephalopathy with reduced diffusion in the bilateral hemispheres. AJNR Am J Neuroradiol 2009;30:825-30 CrossRef Medline

10. Ozutemiz C, Roshan SK, Kroll NJ, et al. Acute toxic leukoencephalopathy: etiologies, imaging findings, and outcomes in 101 patients. AJNR Am J Neuroradiol 2019;40:267-75 CrossRef Medline

11. Tsubouchi Y, Itamura S, Saito Y, et al. Use of high b value diffusionweighted magnetic resonance imaging in acute encephalopathy/encephalitis during childhood. Brain Dev 2018;40:116-25 CrossRef Medline

12. Le Bras A, Proisy M, Kuchenbuch M, et al. Reversible lesions of the corpus callosum with initially restricted diffusion in a series of Caucasian children. Pediatr Radiol 2018;48:999-1007 CrossRef Medline

13. McKinney AM, Short J, Truwit CL, et al. Posterior reversible encephalopathy syndrome: incidence of atypical regions of involvement and imaging findings. AJR Am J Roentgenol 2007;189:904-12 CrossRef Medline

14. Casey SO, Sampaio RC, Michel E, et al. Posterior reversible encephalopathy syndrome: utility of fluid-attenuated inversion recovery MR imaging in the detection of cortical and subcortical lesions. AJNR Am J Neuroradiol 2000;21:1199-1206 Medline

15. McKinney AM, Kieffer SA, Paylor RT, et al. Acute toxic leukoencephalopathy: potential for reversibility clinically and on MRI with diffusion-weighted and FLAIR imaging. AJR Am J Roentgenol 2009;193:192-206 CrossRef Medline

16. Rana L, Sood D, Chauhan R, et al. MR imaging of hypoxic ischemic encephalopathy: distribution patterns and ADC value correlations. Eur J Radiol Open 2018;5:215-20 CrossRef Medline

17. Liauw L, van Wezel-Meijler G, Veen S, et al. Do apparent diffusion coefficient measurements predict outcome in children with neonatal hypoxic-ischemic encephalopathy? AJNR Am J Neuroradiol 2009;30:264-70 CrossRef Medline

18. Chang $\mathrm{KH}$, Han $\mathrm{MH}$, Kim HS, et al. Delayed encephalopathy after acute carbon monoxide intoxication: MR imaging features and 
distribution of cerebral white matter lesions. Radiology 1992;184: 117-22 CrossRef Medline

19. Crombe A, Alberti N, Gilles M, et al. Extensive acute toxic leukoencephalopathy induced by fludarabine: two months follow-up on brain MRI. J Neuroradiol 2015;42:127-30 CrossRef Medline

20. Trivedi SB, Vesoulis ZA, Rao R, et al. A validated clinical MRI injury scoring system in neonatal hypoxic-ischemic encephalopathy. Pediatr Radiol 2017;47:1491-99 CrossRef Medline

21. Lee MS, McKinney AM, Brace JR, et al. Clinical and imaging features of fludarabine neurotoxicity. J Neuroophthalmol 2010;30:3741 CrossRef Medline

22. Shibutani M, Okeda R. Experimental study on subacute neurotoxicity of methotrexate in cats. Acta Neuropathol 1989;78:291-300 CrossRef Medline

23. Wilasrusmee C, Da Silva M, Singh B, et al. Morphological and biochemical effects of immunosuppressive drugs in a capillary tube assay for endothelial dysfunction. Clin Transplant 2003;17(Suppl 9):6-12 CrossRef Medline
24. Jakkani RK, Krishna Reddy GV, Anasuri S, et al. MRI findings in methotrexate-induced acute toxic leukoencephalopathy. Neurol India 2017;65:1439-40 CrossRef Medline

25. Cerase A, Leonini S, Bellini M, et al. Methadone-induced toxic leukoencephalopathy: diagnosis and follow-up by magnetic resonance imaging including diffusion-weighted imaging and apparent diffusion coefficient maps. J Neuroimaging 2011;21:283-86 CrossRef Medline

26. Capizzano AA, Sanchez A, Moritani T, et al. Hyperammonemic encephalopathy: time course of MRI diffusion changes. Neurology 2012;78:600-01 CrossRef Medline

27. Hayakawa K, Koshino S, Tanda K, et al. Diffusion pseudonormalization and clinical outcome in term neonates with hypoxic-ischemic encephalopathy. Pediatr Radiol 2018;48:865-74 CrossRef Medline

28. Barkovich AJ. Concepts of myelin and myelination in neuroradiology. AJNR Am J Neuroradiol 2000;21:1099-09 Medline

29. Barkovich AJ, Miller SP, Bartha A, et al. MR imaging, MR spectroscopy, and diffusion tensor imaging of sequential studies in neonates with encephalopathy. AJNR Am J Neuroradiol 2006;27:533-47 Medline 